\section{Haploid identification using tropicalized haploid inducer progenies in maize}

\author{
Camila Bastos Ribeiro ${ }^{1^{*}}$, Francielly de Cássia Pereira ${ }^{1}$, \\ Lamartine da Nóbrega Filho', Breno Alvarenga Rezende ${ }^{1}$, Kaio \\ Olímpio das Graças Dias ${ }^{1}$, Guilherme Tomaz Braz ${ }^{1}$, Murilo \\ Candido Ruy ${ }^{1}$, Maria Beatriz Silva ${ }^{1}$, Gisele Cenzi ${ }^{1}$, Vânia Helena \\ Techio $^{1}$ and João Cândido de Souza ${ }^{1}$
}

\begin{abstract}
The aim of this study was to identify maize haploid plants and compare the efficiency of identification of maize haploid plants using the R1-nj morphological marker, plant vigor, flow cytometry, chromosome counting, and microsatellite molecular markers under tropical conditions. We also established a protocol for chromosome duplication in maize haploid plants. Fourteen $S_{0: 1}$ and seven $S_{2: 3}$ haploid inducer progenies were crossed with GNZ9501 in 2012/2013 and 2014/2015, respectively. Through use of the R1-nj trait, we were able to identify 552 putative haploid seeds in 2012/2013 and 260 putative haploid seeds in 2014/2015. Only 1.84\% were true positives according to flow cytometry in 2012/2013. In 2014/2015, 75\% of the putative haploids were true negatives according to molecular markers. Plant vigor had a high proportion of true negatives. Molecular markers and flow cytometry are more efficient in classifying plant ploidy level. Chromosome duplication was efficient in all plants.
\end{abstract}

Key words: Flow cytometry, Zea mays, chromosome counting, chromosome duplication, ploidy level.

\section{INTRODUCTION}

Doubled haploid (DH) technology is widely used in maize (Zea mays) breeding for line development. Many steps are required to obtain a DH line: crosses between the haploid inducer and the donor genotype, identification of the haploid seeds, chromosome duplication of the haploid plants, planting the doubled haploid seeds for self-pollination, and, finally, harvesting seeds of the $\mathrm{DH}$ line. However, each of these steps can be inefficient, especially because there is not enough information about this process under tropical conditions.

A key point for application of in vivo induction on a commercial scale is efficient screening that allows the breeder to differentiate haploid kernels or seedlings from those formed from regular fertilization. The morphological R1-nj (Navajo) marker usually used in inducing haploid seeds exhibits purple color in the endosperm and in the embryo, and it has been the trait most used for haploid discrimination (Chase and Nanda 1965). However, this method is subjective, since it is a morphological and a color trait. Other screening traits can be used, such as radicle color (Chaikam et al. 2016) and radicle length (Rotarenco et al. 2010), plant vigor (Battistelli et al. 2013), and pollen viability
Crop Breeding and Applied Biotechnology 18: $16-23,2018$ Brazilian Society of Plant Breeding. Printed in Brazil http://dx.doi.org/10.1590/198470332018v18n1a3 \title{
.
}


(Couto et al. 2015), among others. Molecular and cytogenetic techniques are very important, such as chromosome counting, flow cytometry, and molecular markers. Chromosomal counting is a simple, though laborious, technique; it has been used for many years to make inferences about plant ploidy. Flow cytometry is a good alternative, because it permits DNA quantification (Battistelli et al. 2013, Couto et al. 2013, Couto et al. 2015). Codominant molecular markers, such as microsatellites (Battistelli et al. 2013, Couto et al. 2013, Couto et al. 2015, Li et al. 2009), allow haploid and diploid plants to be distinguished. Zhao et al. (2013) conducted a study to investigate the mechanism underlying haploid formation. They used fluorescence in situ hybridization (FISH) images to understand haploid induction. However, to the best of our knowledge, there are no reports regarding the use of chromosome counting (Metaphase $\mathrm{C}$ ) for identification of haploids in maize.

Various authors have conducted chromosome duplication in maize haploid seeds, obtaining variable percentages of duplicated chromosomes (Chalyk 2000, Battistelli et al. 2013, Couto et al. 2015). However, information regarding the techniques used to confirm and identify chromosome duplications remains limited. Within this context, the aim of this study was to identify maize haploid plants and compare the efficiency of identification of maize haploid plants using the R1-nj morphological marker, plant vigor, flow cytometry, chromosome counting (Metaphase C), and microsatellite molecular markers under tropical conditions. We also established a protocol for chromosome duplication in maize haploid plants.

\section{MATERIAL AND METHODS}

\section{Experimental area and genetic materials}

The experiments were conducted in the greenhouse of the Department of Biology at the Federal University of Lavras (UFLA), Minas Gerais (MG), Brazil, in 2012/2013 and 2014/2015. Laboratory techniques were conducted in the Department of Biology and Department of Agriculture at UFLA. The Molecular Genetics Laboratory of Embrapa Dairy Cattle, Juiz de Fora, MG, was also used to perform flow cytometry of samples obtained in 2014/2015.

First, fourteen plants were selected from an $\mathrm{S}_{0}$ haploid inducer population derived from crossing the KEMS line (Shatskaya et al. 1994) with other tropical elite lines from the Biology Department at the Federal University of Lavras. In the 2012/2013 season, fourteen $S_{0: 1}$ progenies were crossed with the GNZ9501 commercial single hybrid to obtain haploids. Each $\mathrm{S}_{0: 1}$ progeny had approximately 30 plants. These haploid inducer progenies were used as the female parent, since the haploid inducer system is gymnogenetic. To ensure the synchronicity of flowering time, GNZ9501 seeds were sown on four different dates: November 23 and 30 and December 7 and 14, 2012, at weekly intervals.

The 2013/2014 season was used only to advance one inbreeding generation of the seven best progenies in the

previous harvest, 2012/2013. Thus, the $S_{2: 3}$ generation was obtained. In 2014/2015, a cross field was established containing seven $S_{2: 3}$ progenies and the GNZ9501 single hybrid to obtain haploid seeds from the cross between them. To ensure the synchronicity of flowering time, seeds of the GNZ9501 single hybrid were sown in November on four different dates at weekly intervals. In both seasons, crop practices were conducted as recommended for maize.

Seeds obtained in the crosses involving the GNZ9501 commercial hybrid were evaluated for the haploid induction rate (HIR) according to the R1-nj marker and were considered as putative haploids or putative diploids. Subsequently, all putative haploid seeds were sown in pots in the greenhouse to be evaluated and confirmed as haploids. After germination, plants were evaluated considering plant vigor, amount of DNA according to flow cytometry, chromosome number, and band profile with the use of SSR molecular markers. Putative haploids and putative diploids that were confirmed to be true were categorized as true positives (TP) and true negatives (TN). Subsequently, the TPs were subjected to chromosomal duplication. All these traits were evaluated through use of the methodologies described below.

\section{Haploid identification}

\section{R1-nj (Navajo) morphological marker}

First, the HIR was determined based on the R1-nj marker. Seeds harvested from the nonpigmented hybrid GNZ9501 that were pollinated with the pigmented progenies were visually separated according to the presence of purple staining 
in the endosperm and in the embryo. Seeds with purple endosperm (indicating a regular triploid endosperm resulting from successful fertilization with the inducer pollen) but colorless embryo (indicating a haploid embryo of GNZ9501) were designated as putative haploid seeds as described by Chase and Nanda (1965). To verify the effectiveness of the R1-nj color marker and to account for putative misclassifications (Rober et al. 2005, Prigge et al. 2011), all the haploids detected by the above method were grown in the greenhouse and visually scored for plant vigor. Plant vigor was evaluated at 8 and 20 days after sowing seeds in pots in the greenhouse. Short plants with lower sheets were classified as putative haploids.

\section{Flow cytometry}

The putative haploids were sown in pots in a greenhouse on November 21, 2014 and May 6, 2015. Flow cytometry analysis for haploid confirmation was conducted about 14 days after sowing. For this analysis, 20-30 mg of young leaves from the putative haploid plants were used. Each sample of these plants was compared with young leaves of Pisum sativum (9.09 pg / 2C) as an external reference in 2012/2013 and an internal reference in 2014/2015. The extraction buffer for obtaining the nuclear suspension was LB01 (Dolezel 1997).

The histograms obtained were interpreted in WinMDI 2.8 (2009). The nuclear DNA content (pg) of each sample was estimated comparing the position of the G1 peak of the maize sample and the G1 peak of Pisum sativum. The DNA content of the maize sample was estimated using the following expression:

$$
Q=\left(\frac{E}{S}\right) \times R \text {, in which } Q \text { is the quantity of DNA of the sample }(\mathrm{pg} / 2 \mathrm{C}) ; \mathrm{E} \text { is the position of the } \mathrm{G} 1 \text { peak of the sample; }
$$

$\mathrm{S}$ is the reference position of the G1 peak; $R$ is the reference quantity of DNA $(9.09 \mathrm{pg} / 2 \mathrm{C})$.

\section{Chromosome counting}

The putative haploid plants sown in the greenhouse were used for preparation of mitosis slides (Metaphase C) using root tips. The root tips were placed in a pre-treatment using $0.1 \%$ of colchicine for 5 hours in the refrigerator. Subsequently, they were placed in Carnoy (ethanol-acetic acid, $2: 1$ ) and left at $-20^{\circ} \mathrm{C}$. The mitosis slides were set up by the flame-drying technique (Dong et al. 2000). Enzymatic digestion of the cell wall was performed using Cellulase enzyme at $100 \mathrm{U}$ and Pectinase enzyme at $200 \mathrm{U}$ for 45 minutes. For digestion, the samples were oven dried at $37^{\circ} \mathrm{C}$. The slides were stained with $5 \%$ Giemsa and examined under an optical microscope at $100 x$ magnification. For determination of chromosome number, we considered 20 metaphases in each slide.

\section{DNA molecular marker}

For analysis using SSR, leaf samples of all putative haploids and the parental plants were collected. DNA extraction was performed according to Doyle and Doyle (1987). PCR reactions were carried out using the same pair of primers (SSR BNLG1233) recommended by Couto et al. (2013). Gels were stained using silver nitrate. After checking the polymorphism of the parents, the primer was used to detect maternal haploids. Amplification products were separated by $10 \%$ polyacrylamide gel electrophoresis at $100 \mathrm{~V}$ for 90 minutes.

\section{Chromosome duplication}

The haploid plants obtained in 2012/2013 and identified by flow cytometry were subjected to the chromosome duplication protocol at 28 days after sowing, according to the method suggested by Couto et al. (2013). The protocol used was root immersion in $0.1 \%$ colchicine solution, $0.1 \%$ dimethylsulfoxide (DMSO), and $0.1 \%$ Tween 20 for 6 hours at 20 ${ }^{\circ} \mathrm{C}$. After colchicine treatment, seedlings were transferred to the greenhouse. Approximately 14 days after acclimation, flow cytometry was performed again to verify if chromosome duplication was successful.

\section{RESULSTS AND DISCUSSION}

The average number of seeds of crosses used for determination of the HIR was similar using Pop1-F3 and Pop2-F5 as haploid inducers (Table 1). However, the HIR was different between years, showing an overall mean of $2.48 \%$. Besides that, expression of anthocyanin color was observed to be less intense in the 2014/2015 season. Belicuas et al. (2007) noted that this R1-nj gene has variable expression and incomplete penetrance. Expression of the R1-nj has been related to 
environmental conditions and mainly depends on the genetic background of the donor genotype (Chaikam et al. 2016, Eder and Chalyk 2002). This usually occurs because some germplasm sources have expression of natural anthocyanin in the pericarp tissue (Chaikam et al. 2016). Hence, the type of kernel might have an influence on expression of the R1-nj, for example, in flint or dent kernels. Dent kernels usually express more intense anthocyanin color. Eder and Chalyk (2002) noted that haploid induction in the case of maternal haploids is highly influenced by the type of germplasm used as the donor genotype (female parent). These authors compared haploid induction in 20 genotypes, synthetic populations, single and triple hybrids, which have hard kernels, dent kernels, and both (hard $\mathrm{x}$ dent). They observed that many effects, such as the segregating generation and type of kernel, have an influence on haploid induction. Expression of the R1-nj marker was better in dent genotypes and dent $\mathrm{x}$ hard than in genotypes with hard kernels. In this study, the GNZ9501 hybrid has a semi-hard kernel type, so it has potential to express a good HIR. Furthermore, climate conditions, such as temperature and humidity, especially during pollination, can influence induction. Kebede et al. (2011) observed a higher HIR during Mexican winter than in the summer season. The HIR in the winter was $7.37 \%$, compared to $6.11 \%$ in the summer.
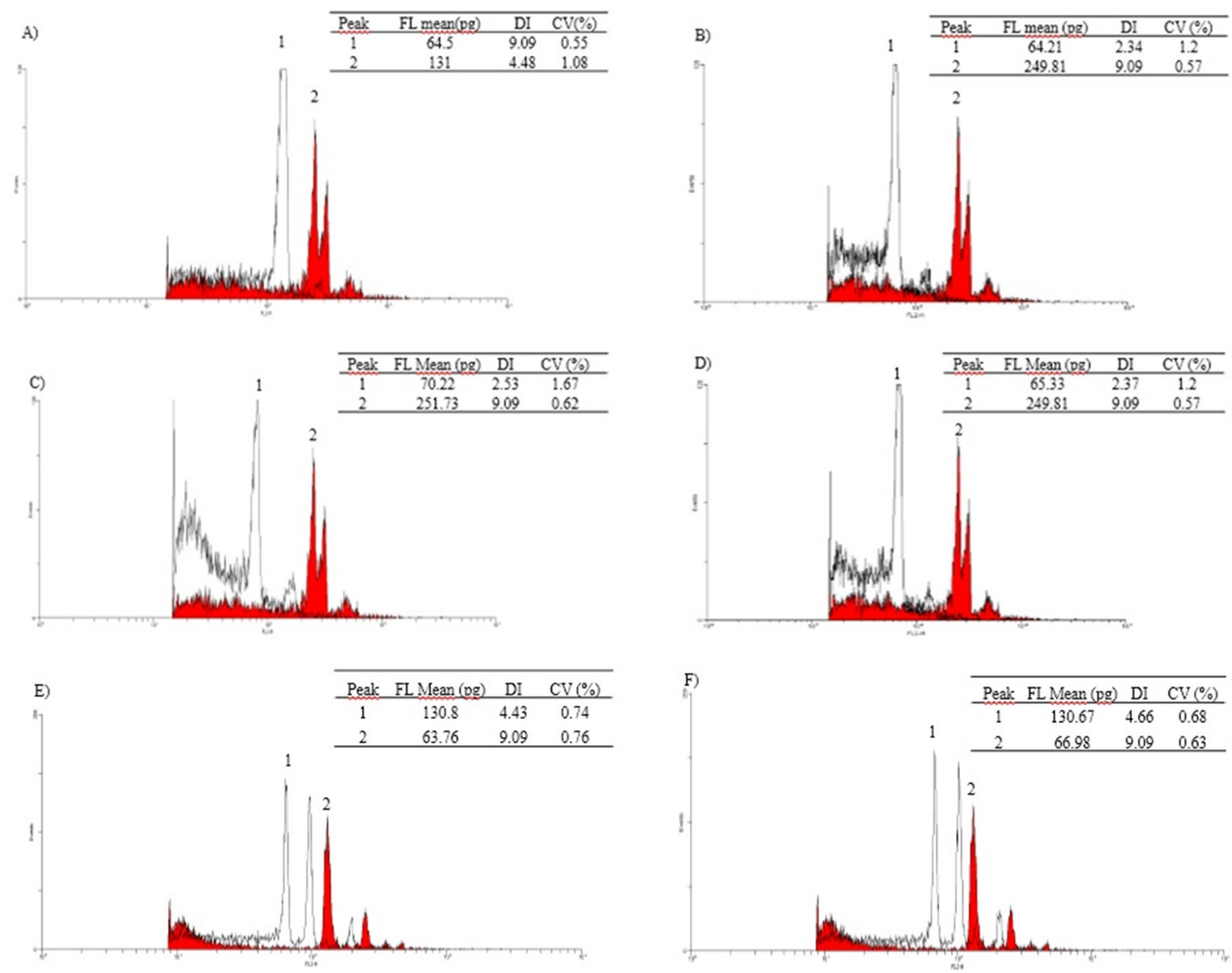

Figure 1. Histograms of the ploidy levels in flow cytometry of the potential haploids selected by R1-nj obtained in 2012/2013 (A, B, C, and D) and in 2014/2015 (E and F). A) Histogram of the diploid female parent, GNZ9501 (peak 1) with Pisum sativum (peak 2). B, C, and D) Histogram of the haploid plant (peak 1) and Pisum sativum (peak 2). E) Histogram of replication 1 of L7 plant (peak 1) and Pisum sativum (peak 2). F) Histogram of replication 2 of L7 plant (peak 1) and Pisum sativum (peak 2). 
Another important observation is that the HIR reduced with tropicalization of the progenies. Progenies were selected based on the HIR. The HIR was 3.14\% in 2012/2013 using fourteen $S_{0: 1}$ haploid inducer progenies. In 2014/2015, this rate decreased to $1.82 \%$ using seven $\mathrm{S}_{2: 3}$ haploid inducer progenies. That means that the HIR showed a $58 \%$ reduction in one generation of selfing in a tropical environment. It is important to note that the progenies used as haploid inducer parents are derived from KEM, which is better adapted to temperate environments (Shatskaya et al. 1994). However, according to Chaikam et al. (2012), CIMMYT has been using temperate inducer lines UH400, RWS, and their hybrid for haploid induction in tropical and subtropical environments in Mexico.

Flow cytometry was performed on all putative haploids obtained in 2012/2013, with selection based on endosperm color. Before this methodology, all 552 seeds of the putative haploids were sown in pots in the greenhouse. The germination percentage was approximately $70 \%$; therefore, flow cytometry was performed on 386 plants. Flow cytometry confirmed three true positives (TP) out of 386 putative haploids evaluated in 2012/2013, all of which are descendants of progeny 6 (Figure 1). Figures $1 \mathrm{~A}, 1 \mathrm{~B}$, and $1 \mathrm{C}$ are examples of histograms derived from haploid plants. The $\mathrm{G} 1$ peak of the haploid is shown in Figure $1(\mathrm{~A})$, in the amount of $2.34 \mathrm{pg} \mathrm{DNA} / 2 \mathrm{C}$. This amount was estimated through comparison with the amount of diploid pea (Pisum sativum) DNA, which has $9.09 \mathrm{pg} / 2 \mathrm{C}$. The smaller peak close to the G1 peak of the sample is regarded as the G2 peak of haploid cells. Flow cytometry has been used in many studies in different species of plants and also in maize DH lines, showing great accuracy, speed, and reliability in identifying them (Couto et al. 2013, Paula et al. 2013).

The selection of haploids in 2014/2015 was also conducted considering plant vigor, in addition to evaluation of the R1-nj (Figure 1E and F). Thus, seedlings with less vigor were considered putative haploids. According to this evaluation, 16 plants were considered true positives (TP). This represents $0.11 \%$ of the HIR or $6 \%$ of the putative haploids previously evaluated according to the R1-nj (Table 2 and 3). Battistelli et al. (2013), working with haploids of maternal origin, found that even in the presence of the anthocyanin inhibitor $\mathrm{Cl}-1$ gene, the R1-nj trait can be replaced by plant vigor. Battistelli et al. (2013) and Prigge et al. (2011) also emphasized that the use of this feature for haploid identification is more effective when a single hybrid is used as the donor genotype, which is the case in this study.

Flow cytometry, chromosome counting, and molecular marker techniques were used to confirm if these 16 plants were TP. However, molecular markers were used in all plants ( 260 plants) to achieve the best sample size. Through flow cytometry analysis, all plants were TN (Figure 1F). Histograms from two replications of the 16 plants analyzed by flow cytometry are in Figure $1 \mathrm{E}$ and F). Furthermore, these diploid plants showed practically the same amount of female donor genotype DNA, which corresponds to a single diploid hybrid. Considering the HIR estimated by flow cytometry, this rate was only $0.02 \%$ in 2012/2013 and $0 \%$ in 2014/2015 (Table 2). Therefore, the efficiency of endosperm color (R1nj) for haploid identification was $1.41 \%$ in $2012 / 2013$ and $0 \%$ in $2014 / 2015$. However, it is important to note that flow cytometry analysis was conducted in 16 low vigor plants, which represents only $4 \%$ of total plants selected as putative haploids by the R1-nj trait. Belicuas et al. (2007) observed $1 \%$ efficiency and Couto et al. (2013) observed $0.86 \%$ efficiency in discriminating haploid plants using the R1-nj. Thus, it is of great importance to study other morphological traits that may allow more efficient ploidy classification.

In addition to flow cytometry, metaphase $C$ slides were obtained to carry out chromosome counting of the putative haploids in 2012/2013 and 2014/2015 (Figure 2). It was observed that both Metaphase C obtained correspond to maize diploid plants. It was not possible to obtain chromosome counting slides of the three TP identified by cytometry in 2012/2013 (Table 2). This probably occurred because these plants have low vigor and, thus, may not have high cell activity. Zhao et al. (2013) conducted a study to investigate the mechanism underlying haploid formation using the

Table 2. Haploid induction rate (HIR in \%) according to the following methodologies: R1-nj, flow cytometry, plant vigor, SSR molecular markers, and chromosome counting by metaphase C, evaluated in 17603 seeds obtained in 2012/2013 and 14270 seeds obtained in 2014/2015 under tropical conditions

\begin{tabular}{lccccc}
\hline Haploid identification & R1-nj & Flow cytometry & Vigor & SSR & Metaphase C \\
\cline { 2 - 6 } Year & \multicolumn{5}{c}{ HIR (\%) } \\
\hline $2012 / 2013$ & 3.14 & 0.02 & - & 0 \\
$2014 / 2015$ & 1.82 & 0 & 0.11 & 0.46 & 0 \\
\hline Overall mean & 2.48 & 0.01 & 0.11 & 0.46 & 0 \\
\hline
\end{tabular}


Table 3. Coincidence of the haploid identification methods in relation to 552 and 260 putative haploids according to R1-nj, evaluated in 2012/2013 and 2014/2015 under tropical conditions

\begin{tabular}{|c|c|c|c|c|}
\hline \multirow[b]{2}{*}{ Year } & Flow cytometry & Vigor & SSR & Metaphase C \\
\hline & \multicolumn{4}{|c|}{ Coincidence (\%) } \\
\hline $2012 / 2013$ & $0.78 \%$ & - & - & 0 \\
\hline $2014 / 2015$ & 0 & 6.15 & 25 & 0 \\
\hline
\end{tabular}

fluorescent in situ hybridization (FISH) technique. However, this is the first study that used chromosome counting as an alternative in identifying haploids in maize.

In 2014/2015, the 260 individuals selected as putative haploids according to the R1-nj were subjected to molecular marker analysis using microsatellite markers (SSR). The polymorphic primer allowed differentiation of homozygous, haploid, and diploid individuals (Figure 3). The gymnogenetic inheritance of the haploid inducer progenies could be confirmed through molecular analyses because the haploids, due to their homozygous nature, showed only one band in the gel, which originated from the hybrid genitor used as the female parent. Plants identified as diploids by flow cytometry also showed two bands using SSR. There were 65 TP representing $0.46 \%$ HIR or $25 \%$ of the total putative haploids characterized by the R1-nj marker (Tables 2 and 3). Other studies also confirmed high efficiency from these markers using the same primer (Battistelli et al. 2013 and Couto et al. 2015). Battistelli et al. (2013) observed 100\% coincidence between SSR and flow cytometry. The 16 plants evaluated by plant vigor and flow cytometry were confirmed as TN by molecular markers (SSR). The results obtained in the present study agree with those of previous studies, indicating that molecular markers are reliable.
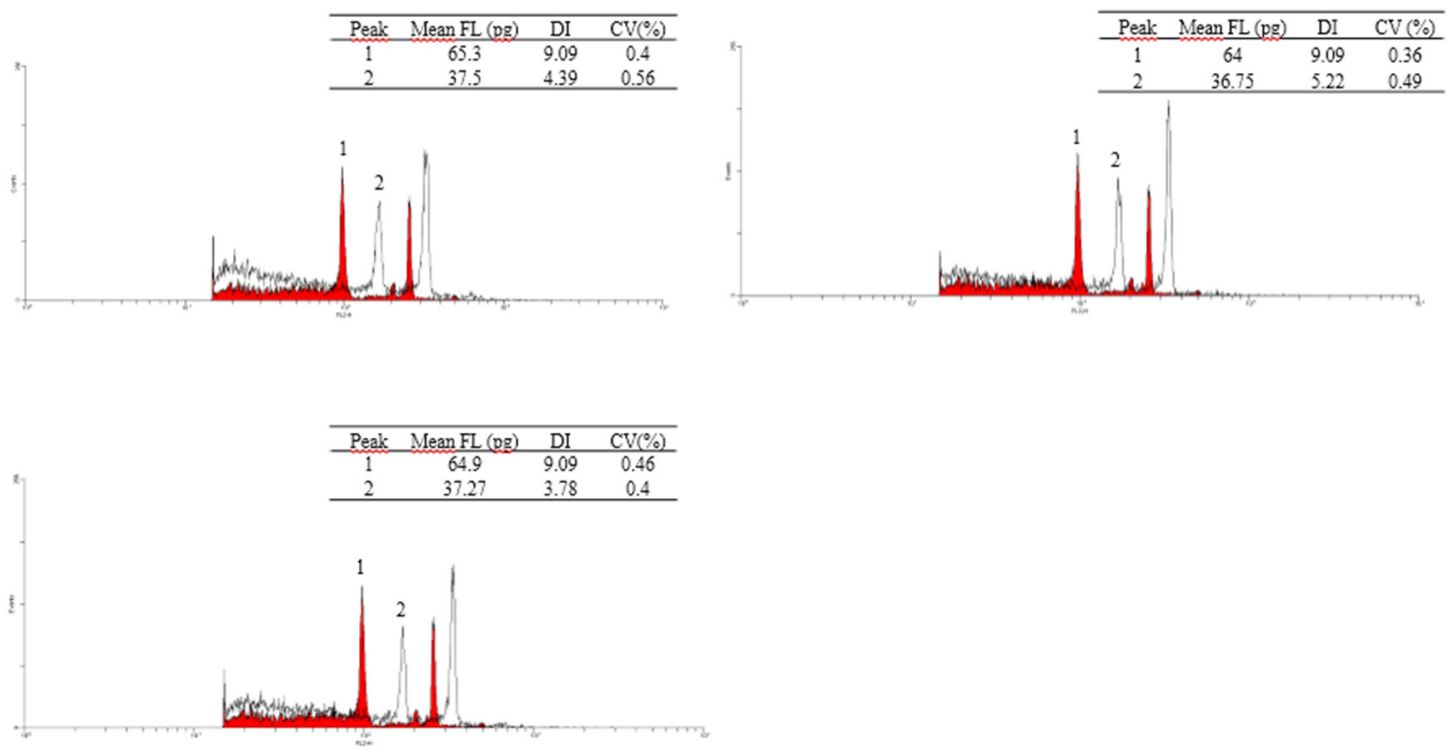

Figure 4. Histograms of ploidy level observed in flow cytometry, evaluated after chromosome duplication of maize plants in $2012 / 2013$. 


\section{CB Ribeiro et al.}

The use of R1- Navajo to identify haploids can be difficult, especially due to its variable expression and incomplete penetrance. Among all methodologies used to confirm ploidy classification in this study, the use of DNA molecular markers and flow cytometry showed greater coincidence of results compared to the R1-nj. The codominant molecular markers allow easy viewing and identification of homozygous and heterozygous individuals. Flow cytometry is also a convenient tool that has been used in many other crops. Obtaining metaphase $\mathrm{C}$ for chromosome counting was not possible for haploid plants as expected.

\section{Chromosome duplication in maize haploids}

Chromosome duplication was carried out in the 3 TP identified by flow cytometry in 2012/2013. The protocol used was suggested by Couto et al. (2013). Flow cytometry was performed 12 days after chromosome duplication to confirm if the duplication protocol was efficient. The histograms obtained showed that duplication was successful in all three haploids (Figure 4). However, further studies are needed to confirm the efficiency of the duplication protocol. After chromosome duplication, plants were transplanted to larger and fertilized pots for self-pollination. However, it was not possible to harvest seeds from these three plants because the inflorescences did not have complete and normal development. This occurrence has been observed in maize DH lines in general. Battistelli et al. (2013) and Couto et al. (2013) evaluated tassel fertility of some DH lines. They observed that approximately $32 \%$ of the plants had viable pollen. These authors reported that $\mathrm{DH}$ lines can show the lowest pollen viability, even in comparison to haploid plants. Dang et al. (2012) highlighted that pollen infeasibility may be pronounced in tropical climates because inducer plants are very sensitive to temperature and photoperiod, especially in the early vegetative stages. Thus, these plants can hinder their development and reproduction. However, it is important to note that self pollination of DH lines has been achieved in the seed industry.

\section{ACKNOWLEDGMENTS}

Research supported by Capes, CNPq, and the Federal University of Lavras.

\section{REFERENCES}

Battistelli GM, Von Pinho RG, Justus A, Couto EG and Balestre M (2013) Production and identification of doubled haploids in tropical maize. Genetics and Molecular Research 12: 4230-4242.

Belicuas PR, Guimarães CT, Paiva LV, Duarte JM, Maluf WR and Paiva E (2007) Androgenetic haploids and SSR markers as tools for the development of tropical maize hybrids. Euphytica 156: 95-102.

Chaikam V, Martinez L, Melchinger AE, Schipprack W and Boddupalli PM (2016) Development and validation of red root marker-based haploid inducers that effectively complement R1-nj (Navajo) marker-based in vivo haploid identification in maize. Crop Science 56: 1-36.

Chaikam V, Mahuku G and Prasanna BM (2012) Design and implementation of maternal haploid induction. In Doubled haploid technology in maize breeding: theory and practice. CIMMYT, Mexico, p. 14-19.

Chase SS and Nanda DK (1965) Comparison of variability in inbred lines and monoploid-derived lines of maize (Zea mays L.). Crop Science 5: $275-276$.

Couto EGO, Davide LMC, Bustamante FO, Von Pinho RG and Silva TN (2013) Identification of haploid maize by flow citometry, morphological and molecular markers. Ciência e Agrotecnologia 37: 25-31.

Couto EGO, Von Pinho EVR, Von Pinho RG, Veija AD, Carvalho MR, Bustamante FO and Nascimento MS (2015) Verification and characterization of chromosome duplication in haploid maize.
Genetics and Molecular Research 14: 6999-7007.

Dang NC, Munsch M, Aulinger I, Renlai W and Stamp P (2012) Inducer line generated double haploid seeds for combined waxy and opaque 2 grain quality in subtropical maize (Zea mays. L.). Euphytica 183: 153-160.

Dolezel J (1997) Application of flow cytometry for the study of plant genomes. Journal of Applied Genetics 38: 285-302.

Dong F, Song J, Naess SK, Helgeson JP, Gebhardt C and Jiang J (2000) Development and applications of a set of chromosome-specific cytogenetic DNA markers in potato. Theorethical Applied Genetics 101: 1001-1007.

Doyle JJ and Doyle JL (1987) A rapid DNA isolation procedure for small quantities of fresh leaf tissue. Phytochemical Bulletin 19: 11-15.

Eder J and Chalyk ST (2002) In vivo haploid induction in maize. Theoretical Applied Genetics 104: 703-708.

Kebede AZ, Dhillon BS, Schipprack W, Araus JL, Banziger M, Semagn K, Alvarado $G$ and Melchinger AE (2011) Effect of source germplasm and season on the in vivo haploid induction rate in tropical maize. Euphytica 180: 219-226.

Li L, Xu X, Jin W and Chen S (2009) Morphological and molecular evidence for DNA introgression in a haploid induction via high oil inducer CAUHOI in maize. Planta 230: 367-376.

Paula CMP, Techio VH, Sobrinho FS and Freitas AS (2013) Distribution 
Haploid identification using tropicalized haploid inducer progenies in maize

pattern of histone $\mathrm{H} 3$ phosphorylation at serine 10 during mitosis and meiosis in Brachiaria species. Journal of Genetics 92: 259-266.

Prigge V, Sánchez C, Dhillon BS and Melchinger AE (2011) Doubled haploids in tropical maize: I. Effects of inducers and source germplasm on in vivo haploid induction rates. Crop Science 51: 1498-1506.

Rober FK, Gordillo GA and Geiger HH (2005) In vivo haploid induction in maize: performance of new inducers and significance for doubled haploid lines in hybrid breeding. Maydica 50: 275-283.
Rotarenco VA, Dicu G, State D and Fuia S (2010) New inducers of maternal haploids in maize. Maize Genetics Cooperation Newsletter 84: 1-7.

Shatskaya OA, Zabirova ER, Shcherbak VS and Chumak MV (1994) Mass induction of maternal haploids in corn. Maize Genetics Cooperation Newsletter 68: 51.

Zhao X, Xu X, Xie H, Chen S and Jin W (2013) Fertilization and uniparental chromosome elimination during crosses with maize haploid inducers. Plant Physiology 163: 721-731. 